\title{
CAMINATA AL AZAR PREDICTIVA DE LA DINÁMICA DE OBESIDAD Y PREDICCIONES DE OBESIDAD Y SOBREPESO EN LA POBLACIÓN INFANTIL DE COLOMBIA Y DE VARIACIÓN PESO/TALLA Y PESO/EDAD EN MÉXICO
}

\author{
Javier Rodríguez'1, Signed Prieto ${ }^{2}$, Esperanza Fajardo 3 , Catalina Correa ${ }^{4}$, Fredy López ${ }^{5}$, \\ JaIro CASTRO ${ }^{6}$, Yolanda SORAcipa ${ }^{7}$.
}

${ }^{1} M D$. Director Grupo Insight. Profesor de la línea de profundización e Internado Especial en Física y Matemática aplicada a la Medicina. Universidad Militar Nueva Granada. - Centro de Investigaciones de la Clínica del Country.

${ }^{2}$ Investigadora Grupo Insight. Universidad Militar Nueva Granada.

- Centro de Investigaciones de la Clínica del Country.

${ }^{3}$ ND. Directora Grupo CATCH. Profesora asociada Facultad de Medicina. Universidad Militar Nueva Granada.

${ }^{4}$ Psicóloga. Investigadora Grupo Insight. Profesora de la línea de profundización e Internado Especial en Física y Matemática aplicada a la Medicina. Universidad Militar Nueva Granada.

- Centro de Investigaciones de la Clínica del Country.

${ }^{5}$ Ingeniero de sistemas. Investigador del Grupo Insight. Universidad Militar Nueva Granada.

${ }^{6} \mathrm{MD}$. Joven Investigador Grupo CATCH. Universidad Militar Nueva Granada.

${ }^{7}$ Lic. Física. Investigadora Grupo Insight. Universidad Militar Nueva Granada.

- Centro de Investigaciones de la Clínica del Country.

Correspondencia: grupoinsight2025@yahoo.es

Recibido: Abril 12 de 2013 Aceptado: Julio 31 de 2013

\begin{abstract}
Resumen
La obesidad y el sobrepeso constituyen problemas de salud pública tanto a nivel mundial como en América Latina. La teoría de la probabilidad y la caminata al azar probabilista han sido base para el desarrollo de predicciones de la dinámica de diferentes epidemias. En este trabajo se estudió el crecimiento de la obesidad en cuatro países con base en la caminata al azar, para confirmar la capacidad de la metodología para describir y predecir esta dinámica específica, y se desarrollaron simulaciones de variables asociadas al estudio de sobrepeso y obesidad en Colombia y México, proyectando una predicción al 2015.
\end{abstract}

Se estudiaron tasas de obesidad de Estados Unidos, Finlandia, Australia e Inglaterra desde 1982 a 1996, en analogía con una caminata al azar probabilista, evaluando su capacidad predictiva para el año 1997. Posteriormente se desarrollaron simulaciones de la dinámica de sobrepeso y obesidad en Colombia con base en reportes reales de los años 2005 y 2010 de acuerdo con el índice de masa corporal (IMC), así como simulaciones de la dinámica de peso/talla y peso/edad en la población mexicana de menores de 0 a 5 años, dividida en cuatro regiones, con base en los reportes de 1999 y 2006, proyectándolas hasta el 2015, con base en la caminata al azar.

El análisis de la dinámica de crecimiento de la obesidad con base en la caminata al azar, permite predecir cuando los índices van a permanecer estables o cuando van a subir en Estados Unidos, Finlandia, Australia e Inglaterra, logrando predicciones con un porcentaje de efectividad igual o superior al 97,85\% para 1997. Las simulaciones obtenidas para México y Colombia predicen un comportamiento probable hasta el 2015, encontrando que en 2015 en Colombia las prevalencias de 
Javier Rodríguez, Signed Prieto, Esperanza Fajardo, Catalina Correa, Fredy López, Jairo Castro, Yolanda Soracipa

obesidad para niños de 0 a 9 años oscilarán entre 4,2 y 11,2, y de sobrepeso para niños entre 10 y 17 años entre 12,9 y 19,4. También se encontraron valores predictivos entre 0,1 y 7,7 para el 2015 en México en niños de 0 a 5 años, respecto a la variable de peso/talla, mientras que respecto a peso/ edad se encontraron entre 0,2 y 11,89 .

Se evidenció que la metodología utilizada es útil para el estudio de diferentes variables asociadas a la dinámica de obesidad y sobrepeso y sirve como base para el desarrollo de predicciones de utilidad en la toma de decisiones de salud pública.

Palabras clave: Probabilidad, caminata al azar, obesidad, sobrepeso.

\title{
PREDICTIVE RANDOM WALK OF OBESITY DYNAMIC AND PREDICTIONS OF OBESITY AND OVERWEIGHT IN CHILDREN FROM COLOMBIA AND WEIGHT/HEIGHT AND WEIGHT/AGE VARIATION IN MEXICO
}

\begin{abstract}
Obesity and overweight are public health problems both globally and in Latin America. The theory of probability and probabilistic random walk have been the basis for developing predictions of the dynamics of different epidemics. In this work, the growth of obesity in four countries is studied based on the random walk, to confirm the ability of the methodology to describe and predict this specific dynamics. Besides, simulations were developed to study variables associated with overweight and obesity in Colombia and Mexico, projecting a possible prediction to 2015.

We studied obesity rates in the United States, Finland, Australia and England from 1982 to 1996 in analogy with a probabilistic random walk, assessing its predictive ability for 1997.Subsequently, dynamics simulations of overweight and obesity in Colombia were developed based on actual reports of 2005 and 2010, as well as simulations of the dynamics of weight/age and weight/ height in the Mexican population younger than 0-5 years, divided into four regions, based on the reports of 1999 and 2006. All the simulations were projected to 2015 , based on the random walk.

The analysis of the dynamics of the obesity rates based on random walk predicts if rates will remain stable or if they will go up in the United States, Finland, Australia and England, making predictions with an effectiveness rate equal to or higher than $97.85 \%$ in 1997 . The simulations obtained for Mexico and Colombia predict a probable behavior until 2015 both with a regular growth and with the random walk. It was found that in 2015 in Colombia the prevalence of obesity for children aged 0 to 9 years will range between 4.2 and 11.2, and overweight in children between 10 and 17 years will range between 12.9 and 19.4. Predictive values between 0.1 and 7.7 were also found in Mexico for 2015 in children aged 0-5 years relating to the variable weight/height, while regarding weight/age values ranging between 0.2 and 11.89 were found.
\end{abstract}

It was evident that the methodology is useful for the study of different variables associated with overweight and obesity dynamics and serve as a basis for developing predictions useful in making public health decisions.

Keywords: probability, random walk, obesity, overweight. 


\title{
CAMINHADA PREVISÕES PREDITIVOS DINÂMICAS ALEATÓRIAS DE OBE- SIDADE E SOBREPESO E OBESIDADE EM CRIANÇAS NA COLÔMBIA E VA- RIAÇÃO DE PESO / ESTATURA E PESO / IDADE NO MÉXICO
}

\begin{abstract}
Resumo
A obesidade e o sobrepeso constituem problemas de saúde pública tanto a nível mundial quanto na América Latina. A teoria da probabilidade e a caminhada ao acaso probabilista têm sido base para o desenvolvimento de predições da dinâmica de diferentes epidemias. Em este trabalho se estudou o crescimento da obesidade em quatro países com base na caminhada ao acaso para confirmar a capacidade da metodologia para descrever e predizer esta dinâmica específica, e se desenvolveram simulações de variáveis associadas ao estudo de sobrepeso e obesidade na Colômbia e no México, projetando uma predição a 2015.

Estudaram-se taxas de obesidade dos Estados Unidos, da Finlândia, da Austrália e da Inglaterra desde 1982 até 1996 em analogia com uma caminhada ao acaso probabilista, avaliando sua capacidade preditiva para o ano 1997. Posteriormente se desenvolveram simulações da dinâmica de sobrepeso e obesidadena Colômbia com base em reportes reais dos anos 2005 e 2010 de acordo ao índice de massa corporal (IMC), assim como simulações da dinâmica de peso/tamanho e peso/idade na população mexicana de menores de 0 a 5 anos, dividida em quatro regiões, com base nos reportes de 1999 e 2006, projetando-as até 2015 , com base na caminhada ao acaso.

Aanálise da dinâmica de crescimento da obesidade com base na caminhada ao acaso permite predizer quando os índices vão permanecer estáveis ou quando vão subir Nos Estados Unidos, na Finlândia, na Austrália e na Inglaterra, logrando predições com uma percentagem de efetividade igual ou superior ao $97,85 \%$ para 1997. As simulações obtidas para o México e a Colômbia predizem um comportamento provávelaté 2015, encontrando que em 2015 na Colômbia as prevalências de obesidade para crianças de 0 a 9 anos oscilarão entre 4,2 e 11,2, e de sobrepeso para crianças entre 10 e 17 anos entre 12,9 e 19,4. Também se encontraram valores preditivos entre 0,1 e 7,7 para 2015 no México em crianças de 0 a 5 anos, respeito à variável de peso/tamanho, por enquanto com respeito a peso/ idade se encontraram entre 0,2 e 11,89 .
\end{abstract}

Evidenciou-se que a metodologia utilizada é útil para o estudo de diferentes variáveis associadas à dinâmica de obesidade e sobrepeso e serve como base para o desenvolvimento de predições de utilidade na toma de decisões de saúde pública.

Palavras chave: probabilidade, caminhada ao acaso, obesidade, sobrepeso.

\section{Introducción}

El sobrepeso y la obesidad a nivel mundial constituyen una problemática preocupante y en creciente aumento, en especial porque se asocia con otras patologías como diabetes, hipertensión y otras enfermedades cardiovasculares que se encuentran ocupando los primeros lugares de morbilidad y mortalidad en el mundo (1). La población infantil se ha visto tan afectada como la población adulta (2), haciendo necesario que se tenga una proyección clara de su comportamiento en el tiempo, que permita el desarrollo de propuestas y la toma de decisiones en el campo de la salud pública. En el caso de Colombia, se ha observado que el comportamiento de esta trayectoria tiene características similares a la tendencia de aumento en México, en donde se ha venido reportando en los últimos años el mayor nivel de prevalencia de sobrepeso en América Latina (3). 
Una particularidad que ha presentado el estudio de la obesidad infantil, es que no se cuenta con datos de crecimiento anual de la epidemia en la mayoría de los países latinoamericanos. Por ejemplo, en Colombia sólo se cuenta con registros de obesidad y sobrepeso por edad de los años 2005 (4) y 2010 (5). Del mismo modo, en México sólo se cuenta con datos sistemáticos de 1999 (6) y 2006 (7). Este hecho hace necesario el desarrollo de nuevas metodologías que permitan establecer un comportamiento probable de la totalidad de la dinámica, que sea de utilidad para establecer proyecciones a futuro.

Metodologías que aplican teorías físicas y matemáticas para el estudio de diferentes epidemias han logrado predicciones de utilidad en salud pública independientemente de variables causales. En trabajos realizados por Rodríguez y colaboradores, desde la teoría de la probabilidad, se logró predecir el número de casos de malaria (8) y de dengue (9), para el año 2007 en Colombia, con un acierto del $100 \%$. Con base en la aplicación del concepto de entropía y la relación $\mathrm{S} / \mathrm{k}$, también se ha logrado predecir la aparición de brotes de la epidemia de malaria en los municipios de Colombia, logrando predicciones de brotes en rangos de tiempo de tres semanas con una efectividad del 99,86\% (10).

En otro estudio, desde la teoría de la probabilidad y la caminata al azar probabilista se ha estudiado el comportamiento la epidemia de dengue en Colombia, obteniendo predicciones por encima del $90 \%$ de exactitud con respecto a datos de vigilancia epidemiológica registrados (11). La probabilidad se puede definir como una cuantificación de la posibilidad de que ocurra un evento en el futuro, en un rango de valores que van desde 0 hasta 1 , donde 0 es equivalente a una posibilidad nula y 1 equivale a que dicho fenómeno se presentará con toda seguridad. La caminata al azar probabilista permite estudiar fenómenos que exhiben comportamientos complejos e irregulares en el tiempo $(12,13)$. Por ejemplo, en el experimento del lanzamiento de una moneda de forma repetida, la caminata cuantifica la tendencia en el tiempo con respecto al punto de inicio. De esta manera, con la caminata al azar se pueden caracterizar fenómenos probabilistas acotando su dinámica y hallando valores numéricos con los que se puede predecir, en el marco de un rango, cuál será la evolución futura de una trayectoria (8). Para estudiar qué tanto se aleja del eje de inicio una medida determinada en el contexto de un experimento que se repite, se utiliza la desviación media cuadrática que permite inferir si el espacio de probabilidad exhibe un cargamiento de las probabilidad o si, por el contrario, todos sus valores tienen la misma probabilidad de aparecer (12).

En este contexto el objetivo de este trabajo es evaluar la capacidad de la metodología desarrollada por Rodríguez y colaboradores con base en la caminata al azar probabilista para describir, simular y predecir el comportamiento de diferentes variables asociadas a la epidemia de obesidad. Para ello se aplicará al estudio de las tasas de obesidad de cuatro países: Estados Unidos, Finlandia, Australia e Inglaterra. También se establecerán simulaciones y predicciones de la dinámica de obesidad infantil en México y Colombia con base en la caminata al azar probabilista, de posible utilidad para programas de prevención en el campo de la salud pública.

\section{Metodología}

\section{Predicción de la dinámica de la tasa de obesidad en Estados Unidos, Finlandia, Australia e Inglaterra}

Partiendo de la tasa de obesidad (IMC $\geq 30 \mathrm{~kg} / \mathrm{m}^{2}$ ) reportada por en el periodo entre 1982 y 1996 en los países de Estados Unidos, Finlandia, Australia e Inglaterra en la revisión realizada por James (1), se estudió la dinámica de cada uno de estos países en el tiempo como una caminata al azar, basados en una metodología desarrollada previamente por Rodríguez y colaboradores (11), con el fin de realizar una predicción para 1997.

Inicialmente, con base en la ecuación 1 , se establecieron las longitudes de todas las variaciones por año, en donde los valores de $\mathrm{Y}$ corresponden a la tasa anual de obesidad en dichos países, y el valor de la variación del eje $\mathrm{X}$ adquiere un valor igual a cero.

$$
L=\sqrt{\left(X_{0}-X_{0}\right)^{2}+\left(Y_{0}-Y_{1}\right)^{2}} \quad \text { Ecuación } 1
$$

Donde las coordenadas cartesianas

$X_{0}, Y_{0}$ : son las coordenadas para el año inicial.

$X_{0}, Y_{1}$ : son las coordenadas para el año siguiente.

En el espacio de probabilidad, cada variación anual de la longitud $L$ representa un evento. Para la obtención de la probabilidad de cada longitud de una variación anual se utilizó la ecuación 2 , que consiste en la razón 
entre la longitud de dicha variación y la suma del total de las longitudes. A partir de este valor se halló la proporción de cada variación anual tomando como referencia la longitud mínima encontrada.

$$
P(L)=\frac{\text { Longitud variacion anual }}{\text { Total longitudes }}=\frac{L}{T L} \quad \text { Ecuación } 2
$$

Se cuantificó, luego, la probabilidad de la tasa de obesidad para cada año, definida como la división entre la tasa de obesidad para cada año dividido en la sumatoria de la totalidad de tasas evaluadas (ecuación 3):

$$
P(N)=\frac{\text { Tasa de obesidad }}{\text { Total }} \quad \text { Ecuación } 3
$$

Se determinó además si existe un cargamiento en dicha probabilidad, utilizando la ecuación 4 que corresponde a la desviación media cuadrática de la caminata al azar probabilista.

$$
P(R n)=\frac{\text { Tasa de obesidad }}{\text { Total }} \pm \frac{1}{2 \sqrt{N}} \quad \text { Ecuación } 4
$$

Donde $N$ es el número de eventos del espacio muestral.

Posteriormente, se construyó un espacio de probabilidad de cuantifica los últimos tres valores consecutivos de las longitudes halladas con el fin de delimitar la dinámica de la tasa de obesidad. Acto seguido, se calculó el promedio aritmético de estos tres valores como el valor de probabilidad para la longitud para el periodo escogido para predecir la tasa de obesidad, en este caso el año 1997, y finalmente, se remplazó este valor en la ecuación 1 con el fin de obtener una ecuación cuadrática en función de $Y_{1}$, ecuación 5 , teniendo como resultado dos valores predictivos, que delimitan un rango dentro del cual se halla el valor predictivo final.

$$
Y_{\text {(año a predecir })}=\frac{2 Y_{(\text {año anterior })} \pm \sqrt{\left(-2 Y_{(\text {año anterior })}\right)^{2}-4\left\{Y_{(\text {año anterior })}{ }^{2}+\left(X_{0}-X_{0}\right)^{2}-\left[P(L)^{2} \times(T L)^{2}\right]\right\}}}{2} \quad \text { Ecuación } 5
$$

Donde: $P(L)$ corresponde al promedio aritmético de la probabilidad para los 3 últimos periodos, y TL representa la sumatoria de las 3 proporciones de cada variación anual con respecto a la longitud mínima hallada en el espacio de probabilidad construido valores de los tres últimos años.

Con el fin de seleccionar el valor predictivo más probable dentro del rango establecido y de este modo determinar la predicción final, se estableció el promedio entre los dos valores predichos y se construyó un espacio de probabilidad que estudia el comportamiento de aumentos o mantenimientos en la tasa de obesidad en tres años consecutivos, para establecer la predicción final con base en el comportamiento de la dinámica en este período; dado que en ninguna de las dinámicas se presentaron disminuciones, esta posibilidad no fue tenida en cuenta.

\section{Simulaciones predictivas de Colombia y México}

Partiendo de la Encuesta Nacional de la Situación Nutricional en Colombia, realizada en el año 2005 (4) y en el año 2010 (5), se tomaron los datos de prevalencia de obesidad de 0 a 9 años con respecto al IMC, divididos en rangos anuales ( $<12$ meses, 1 año, 2 años, etc.), así como los datos de prevalencia de sobrepeso de 10 a 17 años, divididos de la misma forma. Para el caso de las simulaciones del comportamiento de la epidemia en México, se partió de la Encuesta Nacional de Nutrición realizada en 1999 (6) y de los resultados de nutrición de la ENSANUT del 2006 (7), tomando los datos de peso/edad $>2 z$ y peso/talla $>2 z$ en niños de 0 a 5 años para cuatro regiones: centro, norte, sur y región de Ciudad de México. Estos datos fueron tomados como base para la realización de simulaciones de la dinámica de sobrepeso y obesidad para el período comprendido entre 2005-2015 para el caso de Colombia, y de peso/edad $>2 \mathrm{z}$ y peso/talla $>2 \mathrm{z}$ entre 1999-2015 en el caso de México.

Las simulaciones se realizaron con la forma de una caminata al azar establecida a partir de los valores reales obtenidos para cada uno de los países estudiados. Se escogió la caminata al azar de acuerdo con el comportamiento matemático de cargamiento de las probabilidades, lo que permitió generar secuencias de valores cercanos o incluidos dentro de los rangos definidos de acuerdo con el año inicial y el año final. Se generaron dos simulaciones para la dinámica de 
sobrepeso y obesidad infantil en Colombia de acuerdo con el IMC, dos para la dinámica peso/edad y dos para la dinámica peso/talla en México, con el fin de obtener valores predictivos. La proyección se realizó con base en la suposición de un aumento o disminución de las variables observadas equivalentes a la variación reportada en los años de los cuales se cuenta con reporte. En los casos en los que se presenta una disminución que predice un valor menor a la media, se escogieron valores cercanos a la media para realizar la simulación, dada la poca probabilidad de una disminución de este tipo de acuerdo con las condiciones de crecimiento a nivel mundial de esta problemática.

\section{Resultados}

Predicción de la dinámica de la tasa de obesidad en Estados Unidos, Finlandia, Australia e Inglaterra

Los valores de las longitudes para la tasa anual de obesidad para todos los países evaluados se encontraron entre 0 y 0,875 . La probabilidad de dicha longitud varió entre 0,071 y 0,266. Los valores de la razón de distancias respecto a la longitud mínima encontrada estuvieron entre 0 y 5 . Se encontró que las probabilidades de la tasa de obesidad oscilaron entre 0,040 y 0,096, mientras que los valores de la desviación media cuadrática de estos valores estuvieron entre 0,003 y 0,133 , encontrando que la diferencia entre el valor encontrado y el valor esperado está en un rango de $-0,037$ y 0,037 . Los valores encontrados para esta medida indican que el comportamiento de las dinámicas estudiadas no es equiprobable, sino que evidencia un cargamiento que permite el establecimiento de predicciones para cada país. La tabla 1 presenta los valores obtenidos para Australia, los datos restantes no son mostrados.

Al estudiar el espacio de probabilidad de los últimos tres años y aplicando la ecuación 5 , se encontraron dos valores predictivos para la tasa de obesidad para el año 1997 para los cuatro países estudiados: para Estados Unidos fueron de 27,10 y 28,5; para Australia de 16,45 y 18; para Inglaterra de 16,24 y 17,99 y para Finlandia de 19,13 y 19,47 .

El análisis de las probabilidades de aumentos consecutivos o mantenimiento de los valores de tasas de obesidad, evidenció que en tres de los casos estudiados (Estados Unidos, Australia e Inglaterra) siempre se presentaron aumentos en la tasa de obesidad, lo que indica que la probabilidad de un aumento en los años siguientes es 1 y por tanto debe escogerse el valor predictivo correspondiente al aumento de cada una de estas dinámicas y promediarlo con el promedio ya obtenido con los resultados encontrados en la ecuación cuadrática. Se encontró que para Estados Unidos el valor de la predicción final para el año 1997 fue de 28,15; para Australia de 17,61 y para Inglaterra de 17,55. Estos valores fueron contrastados con los valores reportados por James (1), con el fin de determinar el porcentaje de exactitud de la predicción. Dado que los valores reales corresponden a 28,5, 18 y 17,9 respectivamente para cada país, las predicciones presentan una exactitud de 99,77\% para Estados Unidos, 97,85\% para Australia y $98,11 \%$ para Inglaterra.

En el caso de Finlandia, se encontró que la dinámica aumenta durante el período entre 1982 a 1991, y posteriormente se mantiene estable. Con el fin de hallar la predicción para este país, en el espacio de probabilidad de cuantifica los tres últimos valores consecutivos de las longitudes, se tomaron los dos últimos valores que presentaron variaciones y el valor del primer año en el que se mantiene estable, es decir, se tomaron los datos de 1990, 1991 y 1992. Con base en estos valores se establecieron los dos valores predictivos de la ecuación 5. Al analizar el comportamiento probabilista de los aumentos o de que la dinámica se mantenga en un mismo valor en tres años consecutivos se encontró que frente a la posibilidad de que al presentarse en dos ocasiones un valor estable (EE) la probabilidad de que el valor continúe estable (EEE) es 0,17 , mientras que la probabilidad de que se presente un aumento (EEA) es 0 . Por tal razón, los dos valores predictivos hallados fueron promediados para obtener la predicción final. Se encontró un valor predicho de 19,3, que es el mismo valor real reportado por James (1), lo que significa que se logró una predicción con un porcentaje de exactitud del $100 \%$.

\section{Simulaciones Predictivas de Colombia y México}

Se observó que la dinámica tanto de sobrepeso como de obesidad en Colombia presenta una clara tendencia al aumento en la población infantil, solamente en los menores a 12 meses no se presentó un aumento en la prevalencia de las variables estudiadas, en el intervalo 2005 a 2010, el resto de valores presentaron aumentos entre 0,9 y 4,2 para la prevalencia de obesidad en menores de 9 años, y entre 0,7 y 5,4 en sobrepeso en menores entre 10 y 17 años $(4,5)$. 
En cuanto al comportamiento de la dinámica peso/ talla en México, se encontró que esta presenta tanto disminuciones como aumentos entre 1999 y 2006. La región norte presentó disminuciones para todas las edades, con una variación máxima de 6,8 en menores de 1-3 años, a excepción de los menores de 3-4 años, donde se presentó aumento de 0,2. En la región centro se presentó una disminución de 0,20 en los niños de 3-4 años, y un aumento máximo de 2,40 en los niños de 1-3 años. En la región de Ciudad México se presentó una disminución máxima de 3,70 en menores de 1-3 años y un aumento máximo de 2,10 en los menores a 1 año. La región sur presentó una disminución máxima de 2,1 en los niños de1-3 años y un aumento máximo de 1,3 en los menores a 1 año $(6,7)$.

Algo semejante ocurre con la dinámica peso/edad $>2 z$ en México, donde para la región norte se presentan disminuciones entre 0,5 y 5,6 , excepto en los niños entre 4-5 años, donde se presentó un aumento de 1,1. En la región centro se encuentra una disminución máxima de 2,90 en los menores a 12 meses y un aumento máximo de 1,40 en los menores entre 2 y 3 años. En la región Ciudad de México se encuentra una disminución máxima de -6,7, no se encontraron aumentos, y se encontró un valor que no varió entre ambos años, correspondiente a los menores entre 2 y 3 años. En la región sur se encontró una disminución máxima de 4,10 en los menores a 12 meses y un aumento máximo de 2,10 en menores de 3 a 4 años $(6,7)$.

Con base en las simulaciones realizadas a partir de los valores reales, se obtuvieron valores posibles de la dinámica de sobrepeso y obesidad en el intervalo 2005-2010 y 2010-2015 en Colombia de acuerdo con el IMC, así como de la dinámica de peso/edad $>2 z$ y peso/talla > 2z entre 1999 y 2006 y entre 2006 y 2015 en México. Las simulaciones con base en la caminata al azar para Colombia al ser observadas desde 2006 hasta 2015 presentaron valores de prevalencia que oscilaron entre 2,35 y 19,43. Específicamente variaron entre 2,35 y 11,2 para el sobrepeso de niños de 0 a 9 años, mientras que presentaron valores entre 9,68 y 19,43 para la prevalencia de obesidad en niños de 10-17 años. Para el 2015 en Colombia las prevalencias de obesidad para niños de 0 a 9 años oscilarán entre 4,2 y 11,2 , y de sobrepeso para niños entre 10 y 17 años entre 12,9 y 19,4 .

Por otro lado, al observar el comportamiento de las dinámicas para la totalidad de las regiones en México desde 2000 hasta 2015, se encontró que los valores de las simulaciones desarrolladas para la variación peso/talla presentaron valores entre 2,76 y 10,97 para menores de 1 año; entre 0,2 y 11,89 para niños de 1-3 años; entre 1,3 y 5,2 para niños de 2-3 años; entre 2,77 y 7,4 para niños de 3-4 años y entre 1,43 y 7,96 entre 4-5 años. Para el 2015 se predicen valores de peso/talla para menores de 1 año entre 2,76 y 10,97; para niños de 1-3 años entre 0,2 y 11,89; para niños de 2-3 años entre 2,4 y 4,96 y para niños de 4-5 años entre 1,43 y 7,96 .

En cuanto a la dinámica de peso/edad en México durante el período 2000-2015 para todas las regiones estudiadas las simulaciones predijeron valores entre 0,1 y 7,7 para menores de 1 año; entre 0,3 y 6,56 para niños de 1-3 años; entre 0,2 y 5,7 para niños de 2-3 años; entre 0,44 y 6,8 para niños de 3-4 años y entre 0,1 y 7,3 para niños de 4-5 años. Se predice que los valores de peso/edad en México oscilarán para el 2015 entre 0,5 y 0,67 para menores a 1 año; entre 0,36 y 5,36 para niños entre 1-3 años; entre 0,8 y 5,7 para niños de 2-3 años; entre 3,14 y 6,11 para niños de 3-4 años y entre 0,5 y 5,41 para niños de 4-5 años.

Las tablas 2,3 y 4 presentan tres de las simulaciones predictivas desarrolladas: la tabla 2 corresponde a la dinámica de la prevalencia de sobrepeso y obesidad entre el 2005 y el 2015 en Colombia, mientras que las tablas 3 y 4 corresponden a la dinámica en niños menores de 5 años en México de peso/edad y peso/ talla entre 1999 y el 2015 respectivamente. La figura 1 representa gráficamente el comportamiento de una de las simulaciones construidas para Colombia en el periodo 2005-2015, correspondiente a los datos de la tabla 2. La figura 2 presenta una de las simulaciones de la dinámica de peso/edad > 2z en México desde 1999 hasta 2015, con base en la simulación presentada en la tabla 4.

\section{Discusión}

Este es el primer trabajo en el que predice la dinámica de la tasa de obesidad en Estados Unidos, Australia, Inglaterra y Finlandia, con base en su análisis como una caminata al azar probabilista. Los valores predichos presentan porcentajes de exactitud iguales o superiores a $97,85 \%$, logrando incluso una predicción del $100 \%$ en el caso de Finlandia, evidenciando la capacidad de la metodología desarrollada por Rodríguez y colaboradores (11) para cuantificar el comportamiento de esta 
epidemia específica, y constituyéndola en una herramienta útil en la toma de decisiones de salud pública.

Posteriormente se realizaron simulaciones matemáticas de la prevalencia anual de obesidad y sobrepeso infantil en Colombia en menores de 18 años, y de la dinámica de peso/edad y peso/talla en México en menores de 5 años, con base en la forma de la caminata al azar. La confirmación de la capacidad de la caminata al azar probabilista para caracterizar la dinámica de la tasa de obesidad en Estados Unidos, Australia, Inglaterra y Finlandia evidencia que este tipo de metodología permite caracterizar adecuadamente la forma de la dinámica, permitiendo su predicción, logrando valores predictivos para cada uno de los años de los cuales no se cuenta con información empírica. Al mantener como referencia los valores reportados empíricamente en los reportes que sirven como base a este trabajo (4-7), estas simulaciones predictivas podrían ser de utilidad para lograr una mejor comprensión de la dinámica durante los años en los cuales no se cuenta con información empírica, tanto retrospectiva como prospectivamente.

Esta metodología ha mostrado ser útil simulando comportamientos brownianos (12-14) con cargamientos de la probabilidad. En este sentido, puede servir como base para el desarrollo de predicciones de otras variables asociadas la obesidad y el sobrepeso, constituyendo una herramienta útil para el diseño de estrategias de prevención en el campo de la salud pública para este problema de impacto mundial.

Estas simulaciones están basadas en una forma de pensamiento acausal que se opone a metodologías multicausales convencionalmente aplicadas para estudiar este tipo de fenómenos. Por esta razón no se hacen análisis de la relación entre variables que pueden asociarse al incremento de la obesidad y sobrepeso, sino que se estudia el orden matemático del comportamiento de la dinámica en sí.

Precisamente, la aplicación de teorías físicas y matemáticas a otras áreas de la medicina ha aportado métodos diagnósticos y de apoyo en decisiones terapéuticas que pueden ser de gran utilidad e impacto para la salud mundial. En el campo de la cardiología se han logrado establecer diagnósticos clínicos, para diferenciar objetivamente dinámicas cardiacas normales, agudas y crónicas a partir de la teoría de la entropía proporcional (15). También se logró inferir una ley diagnóstica en el contexto de la teoría de sistemas dinámicos que establece la totalidad de posibles dinámicas desde normalidad hasta enfermedad aguda (16), logrando valores de sensibilidad y especificidad del $100 \%$ al ser comparada con el gold standard (17) y una generalización de la totalidad de posibles arterias coronarias normales y reestenosadas en el contexto de la geometría fractal (18). Metodologías similares se han aplicado para la realización de predicciones en el campo de la inmunología y la biología molecular $(19,20)$. En el campo de la morfofisiología celular, se han realizado metodologías de evaluación diagnóstica de células escamosas del epitelio cervical (21) y de eritrocitos (22), de utilidad para el diagnóstico de la citología y la viabilidad de bolsas para transfusión respectivamente. Finalmente, otras metodologías en el campo de la infectología se han utilizado para el establecimiento de predicciones del valor de las células CD4 necesario para las fases de diagnóstico y tratamiento de la pandemia de $\mathrm{VIH} /$ SIDA a partir de valores del cuadro hemático desde la teoría de conjuntos (23) y probabilidad (24).

\section{Agradecimientos}

Este trabajo es resultado del proyecto MED-1077, financiado por el Fondo de Investigaciones de la Universidad Militar Nueva Granada, por lo cual agradecemos el apoyo de la Institución.

Al Centro de Investigaciones de la Clínica del Country, por el apoyo a los trabajos realizados por el grupo de investigación INSIGHT.

\section{Referencias}

1. James WPT. The epidemiology of obesity: the size of the problem (Review). J Intern Med. 2008; 263: 336-352.

2. Organización Mundial de la Salud. Estrategia Mundial sobre Régimen Alimentario, Actividad física y Salud. Sobrepeso y Obesidad Infantiles [Internet]. 2004 [Acceso enero 15 de 2013]. Disponible en: http://www.who.int/dietphysicalactivity/childhood/es/.

3. Marcos-Daccarett N, Núñez-Rocha G, Salinas-Martínez A, Santos-Ayarszagoitia M, Decanini-Arcaute H. Obesidad como factor de riesgo para trastornos metabólicos en adolescentes mexicanos, 2005. Rev salud pública. 2007; 9(2): 180-193.

4. Ministerio de Protección Social, Instituto Colombiano de Bienestar Familiar, Instituto Nacional de Salud. Salud sexual y reproductiva: Resultados Encuesta Nacional de Demografía y Salud 2005. Bogotá: Profamilia, Instituto Colombiano de Bienestar Familiar, Ministerio de la Protección Social; 2005. p 1-283.

5. Ministerio de Protección Social, Instituto Colombiano de Bienestar Familiar, Instituto Nacional de Salud. Encuesta Nacional de la Situación Nutricional en Colombia 2010 ENSIN. Bogotá: Oficina Asesora de Comunicaciones y Atención al Ciudadano ICBF; 2011. p. 1-509. 
6. Rivera Dommarco J, Shamah Levy T, Villalpando Hernández S, González de Cossío T, Hernández Prado B, Sepúlveda J. Encuesta Nacional de Nutrición 1999. Estado nutricio de niños y mujeres en México. Cuernavaca, Morelos, México: Instituto Nacional de Salud Pública; 2001.

7. Shamah-Levy T, Villalpando-Hernández S, Rivera-Dommarco JA. Resultados de Nutrición de la ENSANUT 2006. Cuernavaca, México: Instituto Nacional de Salud Pública, 2007.

8. Rodríguez J. Dinámica Probabilista Temporal de la Epidemia de Malaria en Colombia. Rev Fac Med. 2009; 17(2): 214-221.

9. Rodríguez J, Vitery S, Puerta G, Muñoz D, Rojas I, Pinilla L, et al. Dinámica probabilista temporal de la epidemia de dengue en Colombia. Revista Cubana de Higiene y Epidemiología. 2011; 49(1): 74-83.

10. Rodríguez J. Método para la predicción de la dinámica temporal de la malaria en los municipios de Colombia. Rev Panam Salud Pública. 2010; 27(3): 211-218.

11. Rodríguez J, Correa C. Predicción Temporal de la Epidemia de Dengue en Colombia: Dinámica Probabilista de la Epidemia. Rev salud pública. 2009; 11(3): 443-453.

12. Feynman RP, Leighton RB, Sands M. Probabilidad. En: Feynman RP, Leighton RB, Sands M. Física. Vol. 1. Wilmington: Addison-Wesley Iberoamericana, S. A; 1964. p. 6-1, 6-16.

13. Wiener N. Nonlinear problems in random theory. Cambridge: Technology Press; 1958.

14. Lavenda B. El Movimiento Browniano. En: A. Fernández-Rañada. Orden y Caos. Scientific American. Barcelona: Prensa Científica S.A.; 1990. p. 28 - 37.

15. Rodríguez J. Entropía proporcional de los sistemas dinámicos cardiacos. Predicciones físicas y matemáticas de la dinámica cardiaca de aplicación clínica. Rev Colomb Cardiol. 2010; 17(3).

16. Rodríguez J. Mathematical law of chaotic cardiac dynamic:
Predictions of clinic application. J Med Med Sci. 2011; 2(8): 1050-1059.

17. Rodríguez J, Correa C, Melo M, Domínguez, D, Prieto S, Cardona DM, et al. Chaotic cardiac law: Developing predictions of clinical application. J Med Med Sci. 2013; 4:79-84.

18. Rodriguez J, Prieto S, Correa C, Bernal P, Puerta G, Vitery S, et al. Theoretical generalization of normal and sick coronary arteries with fractal dimensions and the arterial intrinsic mathematical harmony. BMC Medical Physics. 2010; 10:1-6.

19. Rodríguez J, Bernal P, Prieto S, Correa C . Teoría de péptidos de alta unión de malaria al glóbulo rojo. Predicciones teóricas de nuevos péptidos de unión y mutaciones teóricas predictivas de aminoácidos críticos. Inmunología. 2010; 29(1): 7-19.

20. Rodríguez J. Teoría de unión al HLA clase II teorías de Probabilidad Combinatoria y Entropía aplicadas a secuencias peptídicas. Inmunología. 2008; 27(4): 151-166.

21. Rodríguez J, Prieto S, Correa C, Posso H, Bernal P, Vitery S, et al. Generalización fractal de células preneoplásicas y cancerígenas del epitelio escamoso cervical de aplicación clínica. Rev Fac Med. 2010; 18(2): 173-181.

22. Correa C, Rodríguez J, Prieto S, Álvarez L, Ospino B, Munévar A, et al. Geometric diagnosis of erythrocyte morphophysiology: Geometric diagnosis of erythrocyte. J Med Med Sci. 2012; 3(11): 715-720.

23. Rodríguez J, Prieto S, Bernal P, Pérez C, Correa C, Vitery S. Teoría de conjuntos aplicada a poblaciones de leucocitos, linfocitos y CD4 de pacientes con VIH. Predicción de linfocitos T CD4, de aplicación clínica. Rev Fac Med. 2011; 19(2): 148-156.

24. Rodríguez J, Prieto S, Bernal P, Pérez C, Correa C, Álvarez L, et al. Predicción de la concentración de linfocitos T CD4 en sangre periférica con base en la teoría de la probabilidad. Aplicación clínica en poblaciones de leucocitos, linfocitos y CD4 de pacientes con VIH. Infectio. 2012; 16(1): 15-22. 
Javier Rodríguez, Signed Prieto, Esperanza Fajardo, Catalina Correa, Fredy López, Jairo Castro, Yolanda Soracipa

Tabla 1. Valores para cada año de tasas de obesidad de acuerdo al $I M C \geq 30 \mathrm{~kg} / \mathrm{m}^{2}(\mathrm{~N})$, longitud $(L)$; probabilidad de la longitud $(P(L))$; probabilidad de tasas de obesidad $(P(N))$; desviación media cuadrática $(D M C+; D M C$-) y diferencia entre estos (DMC $+P ; D M C$ - $P)$, para el período 1982-1996 en Australia.

\begin{tabular}{|l|c|c|c|c|c|c|c|c|}
\cline { 2 - 9 } \multicolumn{1}{c|}{} & $\mathbf{N}$ & $\mathbf{L}$ & $\mathbf{P}(\mathbf{N})$ & $\mathbf{P}(\mathbf{L})$ & $\mathbf{D M C}+$ & $\mathbf{D M C}-$ & $\mathbf{D M C}+\mathbf{P}$ & $\mathbf{D M C}-\mathbf{P}$ \\
\hline 1982 & 9,0 & & 0,048 & & 0,085 & 0,011 & 0,037 & $-0,037$ \\
\hline 1983 & 9,4 & 0,4 & 0,050 & 0,122 & 0,087 & 0,014 & 0,037 & $-0,037$ \\
\hline 1984 & 9,8 & 0,4 & 0,052 & 0,122 & 0,089 & 0,016 & 0,037 & $-0,037$ \\
\hline 1985 & 10,2 & 0,4 & 0,054 & 0,122 & 0,091 & 0,018 & 0,037 & $-0,037$ \\
\hline 1986 & 10,6 & 0,4 & 0,057 & 0,122 & 0,093 & 0,020 & 0,037 & $-0,037$ \\
\hline 1987 & 11,0 & 0,4 & 0,059 & 0,122 & 0,095 & 0,022 & 0,037 & $-0,037$ \\
\hline 1988 & 11,4 & 0,4 & 0,061 & 0,122 & 0,097 & 0,024 & 0,037 & $-0,037$ \\
\hline 1989 & 11,8 & 0,4 & 0,063 & 0,122 & 0,099 & 0,026 & 0,037 & $-0,037$ \\
\hline 1990 & 12,6 & 0,775 & 0,067 & 0,236 & 0,104 & 0,031 & 0,037 & $-0,037$ \\
\hline 1991 & 13,4 & 0,775 & 0,071 & 0,236 & 0,108 & 0,035 & 0,037 & $-0,037$ \\
\hline 1992 & 14,1 & 0,775 & 0,075 & 0,236 & 0,112 & 0,039 & 0,037 & $-0,037$ \\
\hline 1993 & 14,9 & 0,775 & 0,079 & 0,236 & 0,116 & 0,043 & 0,037 & $-0,037$ \\
\hline 1994 & 15,7 & 0,775 & 0,084 & 0,236 & 0,120 & 0,047 & 0,037 & $-0,037$ \\
\hline 1995 & 16,5 & 0,775 & 0,088 & 0,236 & 0,124 & 0,051 & 0,037 & $-0,037$ \\
\hline 1996 & 17,2 & 0,775 & 0,092 & 0,236 & 0,128 & 0,055 & 0,037 & $-0,037$ \\
\hline
\end{tabular}

Tabla 2. Primera simulación de la dinámica de la prevalencia de sobrepeso y obesidad según el IMC entre el 2005 y el 2015 en Colombia basada en la dinámica de una caminata al azar. Los valores sombreados corresponden a valores reales. El valor en cursiva corresponde a un valor también simulado, debido a que no se cuenta con el valor real de obesidad para niños de 2 años para el 2005.

\begin{tabular}{|c|c|c|c|c|c|c|c|c|c|c|c|c|}
\hline & Edad & 2005 & 2006 & 2007 & 2008 & 2009 & 2010 & 2011 & 2012 & 2013 & 2014 & 2015 \\
\hline \multirow{12}{*}{ Obesidad } & $>12$ meses & 5,6 & 5,3 & 5,38 & 5,15 & 5,1 & 4,9 & 4,45 & 4,82 & 4,44 & 4,26 & 4,2 \\
\hline & 1 año & 2,8 & 3,51 & 4,69 & 5,07 & 6,48 & 7 & 6,96 & 8,12 & 9,75 & 11,1 & 11,2 \\
\hline & 2 años & 2,7 & 2,6 & 2,9 & 3,8 & 4,3 & 4,5 & 5,48 & 6,12 & 5,81 & 6,11 & 6,3 \\
\hline & 3 años & 2,6 & 2,45 & 3,8 & 3,2 & 5 & 5,6 & 5,9 & 6,2 & 8,3 & 7,9 & 8,6 \\
\hline & 4 años & 2,5 & 2,96 & 2,87 & 3,75 & 3,91 & 4,2 & 4,97 & 4,45 & 5,47 & 5,87 & 5,9 \\
\hline & 0-4 años & 3,1 & 3,81 & 3,69 & 4,78 & 4,97 & 5,2 & 5,56 & 5,87 & 6,76 & 7,24 & 7,3 \\
\hline & 5 años & 2,4 & 2,35 & 2,88 & 2,76 & 3,25 & 3,6 & 3,94 & 3,58 & 4,56 & 4,76 & 4,8 \\
\hline & 6 años & 6 & 5,91 & 6,3 & 5,67 & 5,71 & 5,7 & 5,41 & 5,85 & 5,25 & 5,55 & 5,4 \\
\hline & 7 años & 3,2 & 4,01 & 3,82 & 5,55 & 5,83 & 6,1 & 7,34 & 7,07 & 7,68 & 8,89 & 9 \\
\hline & 8 años & 3 & 2,76 & 3,59 & 3,47 & 5,26 & 5,1 & 5,76 & 5,41 & 6,14 & 6,74 & 7,2 \\
\hline & 9 años & 2,4 & 2,35 & 3,11 & 4,78 & 4,99 & 5,2 & 6,78 & 6,13 & 7,46 & 7,87 & 8 \\
\hline & 5-9años & 4,3 & 4,25 & 4,56 & 4,97 & 5,23 & 5,2 & 5,13 & 5,63 & 6,0 & 5,91 & 6,1 \\
\hline \multirow{8}{*}{ Sobrepeso } & 10 años & 10,2 & 11,76 & 11,01 & 13,65 & 14,1 & 14,6 & 15,48 & 16,94 & 17,76 & 18,99 & 19 \\
\hline & 11 años & 10,9 & 11,01 & 12,13 & 13,06 & 13,78 & 13,6 & 14,81 & 14,13 & 15,83 & 16,51 & 16,3 \\
\hline & 12 años & 8,6 & 10,03 & 10,88 & 13,67 & 14,03 & 14 & 13,80 & 15,96 & 18,43 & 19,43 & 19,4 \\
\hline & 13 años & 10,3 & 11,67 & 12,34 & 12,76 & 13,91 & 14,2 & 14,76 & 15,35 & 16,98 & 17,96 & 18,1 \\
\hline & 14 años & 11,5 & 11,8 & 11,62 & 11,97 & 12,16 & 12,2 & 11,87 & 12,52 & 12,66 & 12,83 & 12,9 \\
\hline & 15 años & 9,8 & 9,68 & 10,24 & 11,06 & 11,29 & 11,4 & 11,87 & 12,04 & 12,68 & 13,09 & 13 \\
\hline & 16 años & 11,2 & 11 & 12,01 & 13,02 & 13,09 & 13,8 & 14,99 & 14,44 & 15,61 & 15,99 & 16,4 \\
\hline & 17 años & 10,2 & 10,54 & 10,87 & 10,5 & 11,26 & 12 & 12,34 & 13,26 & 12,11 & 13,76 & 13,8 \\
\hline
\end{tabular}


CAMINATA AL AZAR PREDICTIVA DE LA DINÁMICA DE OBESIDAD Y PREDICCIONES DE OBESIDAD Y SOBREPESO EN LA POBLACIÓN INFANTIL DE COLOMBIA Y DE VARIACIÓN PESO/TALLA Y PESO/EDAD EN MÉXICO

Tabla 3. Primera simulación de la dinámica de peso/talla $>2 z$ en cuatro regiones en México basada en la dinámica de una caminata al azar.

\begin{tabular}{|c|c|c|c|c|c|c|c|c|c|c|c|c|c|c|c|c|c|c|}
\hline Región & Edad & 1999 & 2000 & 2001 & 2002 & 2003 & 2004 & 2005 & 2006 & 2007 & 2008 & 2009 & 2010 & 2011 & 2012 & 2013 & 2014 & 2015 \\
\hline \multirow{5}{*}{ Norte } & $>12$ meses & 10,3 & 9,5 & 10,1 & 8,9 & 8,6 & 8,9 & 7,5 & 7 & 5,4 & 6,3 & 5,1 & 4,3 & 2,9 & 3,5 & 3,1 & 2,8 & 2,76 \\
\hline & 1 a 3 años & 10,4 & 9,1 & 8,3 & 6,4 & 6,9 & 4,5 & 3,8 & 3,6 & 2,1 & 3,3 & 1,7 & 1 & 1,4 & 1,7 & 0,8 & 0,4 & 0,2 \\
\hline & 2 a 3 años & 5,2 & 5,2 & 4,6 & 5,1 & 4,8 & 5 & 4,9 & 4,7 & 4,2 & 4,6 & 4,2 & 4,4 & 4,1 & 4,3 & 4,2 & 4,3 & 4,06 \\
\hline & 3 a 4 años & 5,3 & 5,5 & 5,2 & 5,1 & 5,4 & 5,1 & 5,3 & 5,5 & 5,5 & 5,3 & 5,6 & 5,2 & 5,5 & 5,2 & 5,8 & 5,6 & 5,76 \\
\hline & $4-5$ años & 5,7 & 5,2 & 5 & 4,5 & 4,9 & 5,3 & 5 & 4,7 & 3,9 & 4,5 & 4,7 & 4,3 & 3,6 & 3,9 & 3,5 & 3,7 & 3,41 \\
\hline \multirow{5}{*}{ Centro } & $>12$ meses & 4,2 & 4,8 & 4,1 & 4,3 & 4,7 & 4,6 & 4,8 & 5 & 5,3 & 5,8 & 5,3 & 5,7 & 5,9 & 5,6 & 5,5 & 5,8 & 6,03 \\
\hline & 1 a 3 años & 6,4 & 6,7 & 7,5 & 6,6 & 7,8 & 8,5 & 8,9 & 8,8 & 8,1 & 9,1 & 8,5 & 9,6 & 10,3 & 11,5 & 11,3 & 11,6 & 11,89 \\
\hline & 2 a 3 años & 2,3 & 2,3 & 3,1 & 2,6 & 2,5 & 2,9 & 3,1 & 3,2 & 3,1 & 3,4 & 3,6 & 3,9 & 3,5 & 4,1 & 4,4 & 4,2 & 4,36 \\
\hline & 3 a 4 años & 5,3 & 5,4 & 5,1 & 5,3 & 5,2 & 5 & 5,2 & 5,1 & 5 & 4,6 & 4,9 & 4,6 & 4,8 & 5 & 4,7 & 4,9 & 4,84 \\
\hline & 4 - 5 años & 4,3 & 5 & 4,9 & 4,4 & 4,8 & 5,7 & 5,5 & 5,9 & 6,6 & 6,2 & 6,7 & 6,9 & 7,3 & 7,7 & 7,4 & 7,6 & 7,96 \\
\hline \multirow{5}{*}{$\begin{array}{l}\text { Ciudad } \\
\text { de } \\
\text { México }\end{array}$} & $>12$ meses & 5,1 & 6,3 & 7,1 & 6,1 & 5,5 & 5,9 & 6,7 & 7,2 & 8,2 & 7,3 & 7,6 & 8 & 8,6 & 8,9 & 9,5 & 9,7 & 9,90 \\
\hline & 1 a 3 años & 8 & 7,3 & 5,1 & 4,6 & 4,9 & 4,5 & 4,2 & 4,3 & 3,5 & 2,1 & 3,7 & 1,5 & 1,9 & 0,5 & 1,2 & 0,7 & 0,3 \\
\hline & 2 a 3 años & 1,3 & 1,7 & 2,5 & 1,3 & 2,6 & 3 & 2,7 & 2,9 & 3,5 & 4,3 & 4,7 & 4,4 & 4,8 & 4,5 & 4,2 & 4,7 & 4,96 \\
\hline & 3 a 4 años & 7,8 & 7 & 7,4 & 5,6 & 6,1 & 5,9 & 6 & 5,6 & 4,1 & 5,3 & 4,6 & 3,6 & 3,9 & 3,2 & 3,5 & 2,9 & 2,77 \\
\hline & 4 - 5 años & 4,4 & 3,4 & 3,8 & 3,5 & 3,1 & 3,5 & 3,2 & 3,1 & 2,5 & 3 & 2,7 & 2,2 & 2,1 & 1,6 & 1,9 & 1,7 & 1,43 \\
\hline \multirow{5}{*}{ Sur } & $>12$ meses & 8 & 8,7 & 8,3 & 9 & 8,4 & 8,7 & 8,9 & 9,3 & 9,5 & 9,9 & 9,6 & 10,4 & 10,1 & 9,5 & 10,3 & 10,6 & 10,97 \\
\hline & 1 a 3 años & 5,3 & 5,2 & 5,3 & 4,5 & 3,9 & 3,3 & 3,5 & 3,2 & 2,7 & 1,4 & 1,8 & 1,2 & 0,9 & 0,6 & 1 & 0,6 & 0,5 \\
\hline & 2 a 3 años & 5,6 & 5,1 & 5 & 4,1 & 4,3 & 4,5 & 4,3 & 4,2 & 3,8 & 4 & 3,5 & 3,7 & 3,1 & 2,6 & 2,8 & 2,6 & 2,4 \\
\hline & 3 a 4 años & 4,2 & 4,6 & 4,9 & 4,5 & 5 & 4,7 & 5 & 5,3 & 5,1 & 5,7 & 5,4 & 5,9 & 6,5 & 5,9 & 6,6 & 6,4 & 6,714 \\
\hline & 4 - 5 años & 4,5 & 5,1 & 4,7 & 4,6 & 5,1 & 4,8 & 5 & 5,2 & 5,3 & 5,9 & 5,5 & 5,7 & 5,4 & 5,4 & 5,8 & 6,2 & 6,1 \\
\hline
\end{tabular}

Tabla 4. Primera simulación de la dinámica de peso/edad $>2 z$ en cuatro regiones en México basada en la dinámica de una caminata al azar. Los valores en cursiva corresponden a valores simulados, debido a que no se cuenta con valores reales para los menores de 1-3 y 3-4 años en la región Ciudad de México para el 2006.

\begin{tabular}{|c|c|c|c|c|c|c|c|c|c|c|c|c|c|c|c|c|c|c|}
\hline Región & Edad & 1999 & 2000 & 2001 & 2002 & 2003 & 2004 & 2005 & 2006 & 2007 & 2008 & 2009 & 2010 & 2011 & 2012 & 2013 & 2014 & 2015 \\
\hline \multirow{5}{*}{ Norte } & 12meses & 7,5 & 7,7 & 5,2 & 6,3 & 3,3 & 1,5 & 2,3 & 1,9 & 1,5 & 0,8 & 1,6 & 1,3 & 2,1 & 2,9 & 2,1 & 0,7 & 0,5 \\
\hline & 1 a 3 años & 6,5 & 6,01 & 6,56 & 6,13 & 6,51 & 5,8 & 6,12 & 6 & 5,6 & 5,8 & 5,4 & 5,71 & 5,9 & 5,57 & 5,7 & 5,43 & 5,36 \\
\hline & 2 a 3 años & 5,3 & 4,5 & 3,2 & 4,3 & 4,6 & 3,9 & 3,78 & 3,6 & 3,36 & 3,6 & 2,7 & 2,5 & 2,1 & 1,9 & 1,6 & 1,7 & 1,41 \\
\hline & 3 a 4 años & 5,2 & 4,87 & 5 & 4,2 & 4,5 & 4,6 & 4,38 & 4,3 & 4,7 & 4,1 & 3,8 & 3,9 & 3,4 & 3,53 & 3,5 & 3,3 & 3,14 \\
\hline & 4 - 5 años & 2,9 & 2,8 & 3,1 & 3,7 & 3,3 & 3,9 & 4,2 & 4 & 4,2 & 4,6 & 4,9 & 4,5 & 5,2 & 4,8 & 5,2 & 5,4 & 5,41 \\
\hline \multirow{5}{*}{ Centro } & $>12$ meses & 5,5 & 4,67 & 5,2 & 3,5 & 2,9 & 3,3 & 3,01 & 2,6 & 2 & 2,3 & 1,5 & 0,6 & 1,4 & 0,4 & 0,8 & 0,3 & 0,13 \\
\hline & 1 a 3 años & 4,9 & 2,8 & 3,5 & 4,6 & 3,9 & 2,96 & 2,71 & 2,6 & 2,4 & 1,2 & 2,1 & 0,5 & 0,7 & 0,3 & 0,8 & 0,4 & 0,36 \\
\hline & 2 a 3 años & 2,5 & 3 & 2,4 & 3,15 & 3,33 & 4,3 & 3,77 & 3,9 & 4,2 & 4,6 & 4 & 4,4 & 5 & 5,5 & 5,2 & 5,4 & 5,7 \\
\hline & 3 a 4 años & 3,6 & 3,3 & 4,2 & 3,9 & 4,3 & 4,76 & 4,57 & 4,7 & 4,7 & 5,2 & 5 & 5,5 & 5,3 & 5,7 & 6 & 5,8 & 6,11 \\
\hline & 4 - 5 años & 4 & 3,9 & 3,2 & 3,86 & 3,4 & 2,7 & 3,35 & 3,2 & 3,3 & 2,8 & 3 & 2,6 & 2,3 & 2,6 & 2,2 & 2,3 & 2,17 \\
\hline \multirow{5}{*}{$\begin{array}{l}\text { Ciudad } \\
\text { de } \\
\text { México }\end{array}$} & $>12$ meses & 6,2 & 5,6 & 5,1 & 5,87 & 4,3 & 3,05 & 3,59 & 3,1 & 2,8 & 3,0 & 1,6 & 1,9 & 1,5 & 0,8 & 1,1 & 1,2 & 0,9 \\
\hline & 1 a 3 años & 4,4 & 2,5 & 3,1 & 2,7 & 1,39 & 0,96 & 0,73 & 3,85 & 3,2 & 3,6 & 3,4 & 3,1 & 3,2 & 3,4 & 3,3 & 3,4 & 3,1 \\
\hline & 2 a 3 años & 0,8 & 1 & 0,7 & 0,8 & 0,6 & 0,5 & 0,9 & 0,8 & 0,6 & 0,9 & 1,2 & 0,5 & 0,8 & 1,0 & 0,2 & 0,6 & 0,8 \\
\hline & 3 a 4 años & 1,6 & 1,54 & 1,87 & 0,54 & 0,97 & 0,44 & 0,93 & 3,67 & 4,0 & 3,8 & 4,3 & 5,1 & 4,7 & 5,6 & 5,1 & 6,1 & 6,3 \\
\hline & 4 - 5 años & 7,5 & 5,4 & 7,3 & 2,4 & 3,7 & 0,4 & 1,1 & 0,8 & 1,3 & 0,6 & 1,0 & 0,7 & 0,5 & 0,2 & 0,1 & 0,4 & 0,5 \\
\hline \multirow{5}{*}{ Sur } & $>12$ meses & 4,7 & 4,91 & 3,3 & 2,4 & 3,6 & 1,27 & 0,9 & 0,6 & 0,8 & 0,1 & 0,4 & 0,3 & 0,9 & 0,3 & 0,5 & 0,9 & 0,67 \\
\hline & 1 a 3 años & 1,6 & 2,3 & 1,9 & 1,4 & 2,2 & 2,11 & 2,9 & 2,4 & 2,1 & 2,6 & 2,4 & 2,2 & 2,9 & 2,7 & 3,5 & 3,2 & 3,43 \\
\hline & 2 a 3 años & 4,7 & 4,99 & 4,34 & 4,45 & 3,7 & 4 & 3,83 & 3,8 & 3,5 & 3 & 2,6 & 3,1 & 3,4 & 2,9 & 2,7 & 2,9 & 2,64 \\
\hline & 3 a 4 años & 2 & 2,9 & 2,66 & 3,6 & 3,1 & 3,95 & 3,82 & 4,1 & 3,7 & 4,2 & 4,5 & 5,4 & 4,9 & 5,7 & 6 & 6,6 & 6,8 \\
\hline & 4 - 5 años & 3,2 & 3,3 & 3,11 & 2,9 & 3,19 & 2,8 & 3,09 & 3 & 3,4 & 2,6 & 2,3 & 2,5 & 2,8 & 3 & 2,7 & 2,6 & 2,74 \\
\hline
\end{tabular}


Javier Rodríguez, Signed Prieto, Esperanza Fajardo, Catalina Correa, Fredy López, Jairo Castro, Yolanda Soracipa

Figura 1. Representación gráfica de la simulación de obesidad en menores de 0 a 9 años y de sobrepeso en menores de 10 a 17 años, correspondientes a la tabla 3.

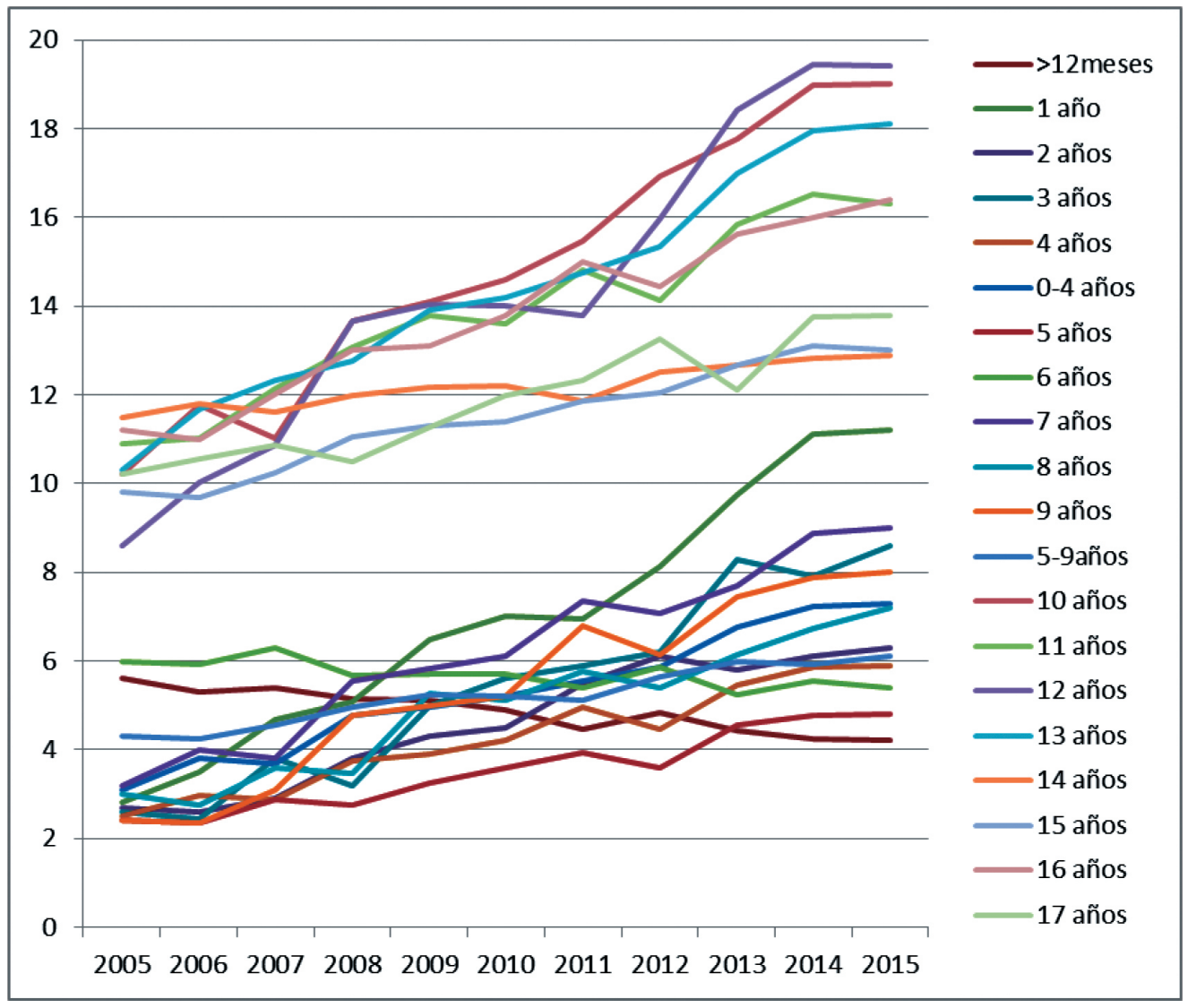

Figura 2. Representación gráfica de la simulación de la dinámica de pesoledad $>2 z$ en el área norte de México basada en la dinámica de una caminata al azar, correspondiente a la tabla 4.

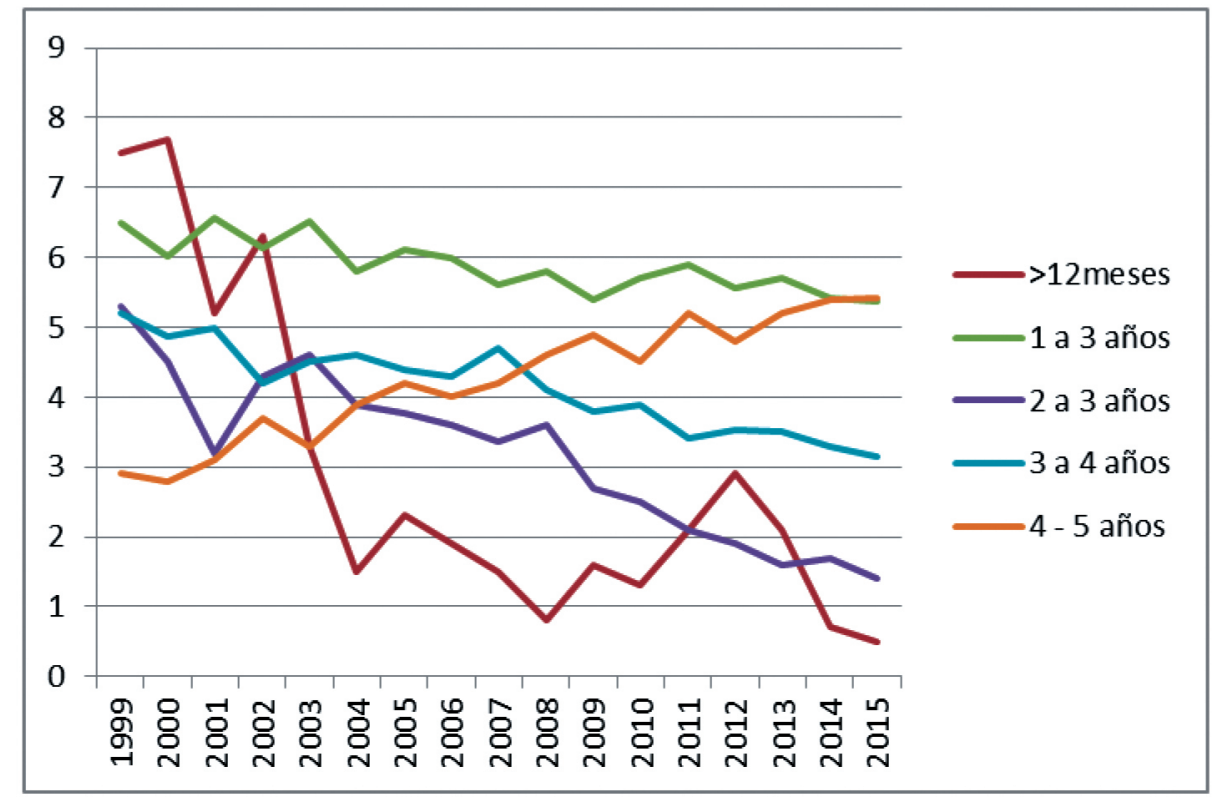

